\title{
Filigrane
}

Écoutes psychanalytiques

\section{Le devenir de la psychanalyse. Que nous disent les pratiques institutionnelles?}

\section{Didier Drieu et Sophie Gilbert}

Volume 24, numéro 2, automne 2015

Le devenir de la psychanalyse. Que nous disent les pratiques institutionnelles?

URI : https://id.erudit.org/iderudit/1036527ar

DOI : https://doi.org/10.7202/1036527ar

Aller au sommaire du numéro

Éditeur(s)

Revue Santé mentale au Québec

ISSN

1192-1412 (imprimé)

1911-4656 (numérique)

Découvrir la revue

Citer ce document

Drieu, D. \& Gilbert, S. (2015). Le devenir de la psychanalyse. Que nous disent les pratiques institutionnelles ? Filigrane, 24(2), 7-11.

https://doi.org/10.7202/1036527ar 


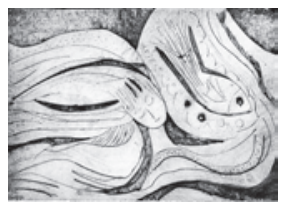

\section{Le devenir de la psychanalyse. Que nous disent les pratiques institutionnelles?}

\section{Didier Drieu et Sophie Gilbert}

$\Lambda$

près un colloque organisé à Caen, en France, intitulé «Fondamentaux de la clinique institutionnelle, les mutations entre hier et aujourd'hui », ainsi que la publication de deux ouvrages collectifs (46 commentaires de textes fondamentaux sur la clinique institutionnelle et Violence et institutions), nous avons répondu à l'invitation de Filigrane de contribuer à la thématique de ses derniers numéros, soit le devenir de la psychanalyse, et ce, sous l'angle spécifique de l'actualité des pratiques institutionnelles actuelles et de l'héritage à partir duquel celles-ci se sont élaborées.

Dans les écrits préalables au colloque, nous revenions sur l'importance de la psychanalyse comme référence majeure dans la mise en place du mouvement institutionnel de l'après-guerre, en parallèle à un engagement sociopolitique et au croisement de pratiques s'appuyant sur les apports spécifiques des sciences humaines (incluant la psychanalyse, mais aussi divers courants de la psychiatrie, l'anthropologie, la philosophie phénoménologique). En témoigne l'expérience de Tosquelles, le fondateur du courant de la psychothérapie institutionnelle qui, après Pinel et Simond, réinvente une forme de désaliénation en associant les patients et le collectif soignant pour penser le soin dans la quotidienneté. Certaines conditions se discernent, afin que l'institution devienne thérapeutique en elle-même: un climat collectif soutenant la fonction d'accueil, la qualité phorique du travail des soignants (observations et mise en sens) et une réflexion sur la constellation transférentielle dans laquelle se retrouvent soignants et patients.

Ce mouvement s'étendra au-delà des projets menés dans quelques lieux phares. À l'époque, beaucoup de psychiatres sont aussi psychanalystes et, notamment avec les infirmiers et les psychologues, ils vont tenter d'articuler plusieurs registres complémentaires tels que le soin, le thérapeutique, l'éducatif et la réhabilitation psychosociale. En découlera l'expérience de 
la sectorisation sous-tendue non pas par la transformation de l'institution (ou de l'organisation) comme dans la psychothérapie institutionnelle, mais plutôt par une présence active des soignants dans l'accueil, au sein de l'établissement et dans l'environnement des patients. Plus encore, il s'agit de construire des alternatives à l'internalisation, considérant que l'hospitalisation de longue durée induit certaines formes de chronicisation. L'objectif est d'ouvrir sur le dedans et le dehors, en instituant des repères dans l'accompagnement des patients dans les différentes phases du soin. Ces démarches ont été initiées par des psychanalystes comme R. Diatkine, S. Lebovici, puis P.-C. Racamier en France, et d'autres comme M. Lemay à Montréal.

Toutefois, cet «esprit du soin» sera contré par des résistances internes à la politique de sectorisation (clivages entre les équipes dans les institutions, alliances défensives entre les médecins, les équipes et l'administration), et plus particulièrement aujourd'hui, par la réticence face aux profondes mutations dans l'organisation de la tâche et de la professionnalité des intervenants. L'important bouleversement actuel des pratiques découle de l'emphase sur la gestion des prestations aux dépens du travail du collectif, et de l'accent mis sur les procédures d'évaluation des risques aux dépens du projet.

Du reste, certaines analyses de ces transformations ou mutations ont su se développer; elles s'inspirent à la fois des travaux de la psychosociologie (tels ceux de E. Enriquez) et de la psychanalyse, ce dont témoigne la référence aux théories de l'intersubjectivité (initiées par Winnicott et Bion) et à l'analyse de groupe (tels que ceux de D. Anzieu, J. C. Rouchy et R. Kaës). En particulier, les travaux de Kaës, à qui l'on doit la conceptualisation d'une réflexion sur «les effets de l'inconscient dans les institutions» (1988) — incluant les institutions soignantes, éducatives et les sociétés de psychanalyse - , se sont avérés porteurs. À la lumière de ces théories, il s'agit de penser les nouages et/ou les espaces traumatiques qui provoquent la déliaison dans les institutions. Cette négativité se dévoile brusquement par les «souffrances» des équipes et des professionnels dans leur exercice clinique. Il y a alors nécessité de «pouvoir analyser la manière dont la clinique peut se trouver organisée (ou souvent malheureusement désorganisée) dans ses différents registres processuels au sein des institutions» (Drieu et al., 2013).

En référence à la psychanalyse, il s'avère possible de considérer les différents espaces psychiques impliqués, incluant la singularité de chaque acteur, mais aussi la dynamique des liens (intersubjectivité), celle du groupe, voire même de la culture (le domaine du transsubjectif), car «selon les cas, les 
problématiques peuvent concerner les enjeux de la fondation (le projet, l'histoire des origines à la refondation par des nouvelles directions), la fonction accueil et les rapports aux sujets accueillis, les professionnels dans leur rapport à leurs statuts et leurs fonctions mais aussi les rapports à l'organisation, de plus en plus marquée par un fonctionnement procédural qui semble prendre la place des hiérarchies pyramidales d'autrefois» (ibid.). Ainsi, l'avenir de la psychanalyse au sein des pratiques institutionnelles serait tributaire d'un mouvement d'extension par rapport à son objet initial, l'intrapsychique, et de la fondation, comme nous y invite R. Kaës (2010), d'une nouvelle approche «métapsychologique des espaces coordonnés».

Notre dossier s'ouvre donc sur l'actualité de la clinique institutionnelle en France. Didier Drieu et Pascal Crété insistent sur la fonction de l'accueil, dans le travail clinique institutionnel, qui doit pouvoir être porté par le collectif soignant. En ce sens, il est essentiel de développer la dimension phorique (observations/élaboration dans le partage/transformation) de ces collectifs, un travail souvent mis à mal de nos jours, puisque peu soutenu dans son architecture (ses méta-cadres) par l'organisation institutionnelle.

L'article de Jean-Yves Chagnon, qui porte sur le $13^{\mathrm{e}}$ arrondissement, retrace l'histoire particulière de la naissance de la pédopsychiatrie française dans les années d'après-guerre. En suivant l'itinéraire particulier de R. Diatkine, le lecteur est invité à redécouvrir les repères soutenant l'accueil et les médiations thérapeutiques dans les centres de jour pour enfants. Comment soutenir, par la présence soignante, le plaisir d'investir, de "désirer», ou même de "fonctionner», là où règne chez l'enfant le désinvestissement voire l'inhibition? Cette question est particulièrement prégnante au moment où la pédopsychiatrie, en France comme au Québec, est de plus en plus ébranlée dans ses fondations (emphase sur l'expertise) et débordée par des demandes de soins psychiques adressées dans l'urgence.

Dans la lignée de l'expérience du $13^{e}$ arrondissement, Guillemette Balsan témoigne de l'apport de la psychanalyse dans les prises en charge hospitalières d'adolescents. Initiés par H. Flavigy, les soins psychiatriques à l'Institut Mutualiste Montsouris auprès d'adolescents souffrant de troubles du comportement alimentaire s'inspirent de la psychanalyse, en particulier d'une métapsychologie des processus psychiques propres à l'adolescence. À la lumière du processus de séparation/différenciation, l'auteur prône l'importance d'une prise en charge plurifocale. Cependant, en ce qui concerne les patients anorexiques cachectiques, il va de plus en plus s'inspirer des théories de l'attachement et de la mentalisation. Puis, l'auteur évoque la 
possibilité d'accueillir l'informe et de travailler à la réanimation des autoérotismes dans une forme de compagnonnage institutionnel.

Dans un autre contexte, celui du Brésil, Laís Macêdo Vilas Boas, Deise Matos Do Amparo, Sandra Francesca Conte de Almeida et Katia Cristina Tarouquella R. Brasil. abordent la clinique des adolescents en conflit avec la loi sous l'angle de dispositifs d'intervention savamment articulés entre des psychanalystes, des étudiants et des éducateurs. Il s'agit de repenser le cadre d'intervention dans ses fondements, afin de permettre une élaboration de l'angoisse de l'adolescent. S'appuyant sur les notions de demande, de dynamique transférentielle, de cadre, et de symbolisation, les auteurs présentent un cas clinique richement documenté illustrant les aléas du travail psychanalytique en institution auprès d'adolescents ayant manifesté des agirs violents. Pour l'analyste, s'il faut tenir compte de la demande sociale, incluant celle de l'institution, il faut aussi pouvoir s'en différencier en impliquant le sujet adolescent dans ses actes, son discours, voire son désir.

Julie Achim et Miguel Terradas, en proposant une réflexion sur l'apport de la mentalisation dans la pratique clinique en pédopsychiatrie, prolongent cette élaboration sur le devenir de la psychanalyse dans la clinique institutionnelle. Plus précisément, les auteurs s'intéressent aux enfants et/ou adolescents repliés dans des troubles des conduites ou des agirs, de même qu'aux problèmes posés par la ré-organisation des soins, provoquant des risques d'escalade dans la médication aux dépens du travail thérapeutique. Ils proposent de réviser les pratiques cliniques de façon à établir, auprès des intervenants, des repères quant aux capacités de mentalisation et d'autorégulation.. Ce faisant, il s'agit de mieux évaluer les difficultés et les ressources des enfants et des parents, afin d'orienter le travail thérapeutique et de les amener à s'intéresser tant à leur monde interne qu'à celui d'autrui.

Nadine Proia-Lelouey dans son article sur l'actualité de la psychanalyse dans l'institution du soin, nous invite à questionner la place de la psychanalyse et par extension, les conséquences de celle-ci sur les dispositifs cliniques. Selon l'auteure, la postmodernité et l'hypermodernité provoqueraient de nouveaux paradoxes articulés entre permissivité et responsabilité, ainsi que de nouvelles formes de malêtre suscitant une psychologisation des souffrances sociales. En lien avec le passage de la psychiatrie à la santé mentale, l'institution de soins et les cliniciens risquent de devenir porteurs de nouvelles figures de régulation et de contrôle social. La psychanalyse pourrait résister à ces nouvelles normes à condition de créer des dispositifs propres à étayer un travail d'appropriation subjective. 
Reprenant le questionnement sur les mutations dans les institutions et les nouvelles formes de souffrances psychiques, Jean Pierre Pinel, à partir du modèle de Jean Claude Rouchy, expose de manière synthétique la méthodologie des dispositifs d'intervention clinique en institution. Le dispositif doit s'instaurer à distance du cadre institutionnel habituel et être déterminé par l'analyste (sans pour autant être préfabriqué), de façon à ce que s'opère une forme de métabolisation des différentes réalités psychiques: celle du professionnel dans sa singularité, celle des équipes de travail et celle de l'institution. L'analyste est pris dans des mouvements transférentiels puissants qui vont l'assigner à différentes places comme celle de plus en plus fréquente de «répondant institutionnel», ce qui doit le conduire à un travail sur le méta-cadre avec ses collègues en intervision. Au préalable, il s'agira de bien différencier, dans les demandes qui peuvent être adressées aux intervenants cliniciens, les différentes méthodologies d'intervention, afin de proposer le cadre le plus adéquat pour résister au mieux à la déliaison, et parfois même, au chaos.

Enfin, pour clore ce dossier, nous accueillons la contribution de Vincent Cardinal qui expose la situation de la psychanalyse en institution au Québec, plus précisément dans un service de deuxième ligne adressé aux enfants, aux adolescents et aux familles. Après avoir remis en contexte socioculturel la situation des institutions de soins en santé mentale, et particulièrement, les contraintes imposées aux psychothérapeutes d'orientation psychanalytique, son propos complète les initiatives inspirantes et d'outre-mer qui auront constitué le corps de ce dossier. 